\title{
SEQUÊNCIA GENERALIZADA DE FIBONACCI E RELAÇÕES COM O NÚMERO ÁUREO
}

\author{
Francisco Regis Vieira Alves ${ }^{1}$ \\ Instituto Federal de Educação, Ciência e Tecnologia do Ceará
}

\section{RESUMO}

Neste trabalho apresentamos modelos que generalizam a noção de Sequência de Fibonacci - SF. Neste sentido, com origem numa argumentação recorrente de um curso de graduação, que adota o modelo de produção de coelhos imortais, temos a possibilidade de identificar propriedades generalizadoras vinculadas com a noção de Sequência Generalizada de Fibonacci - SGF, bem como, possibilidades de obtenção do número de ouro e suas variantes.

Palavras-Chave: Fibonacci; Sequência de Fibonacci; Sequência Generalizada de Figonacci.

\section{Introdução}

De modo tradicional, os livros de História da Matemática - HM definem a seguinte sequência recursiva indicada por $\left\{f_{n}\right\}_{n \in I N}$ e descrita do seguinte modo $f_{n}=f_{n-1}+f_{n-2}$, para $n>2$. Por outro lado, a mesma formula pode ainda ser expressa por $f_{n+1}=f_{n}+f_{n-1}$, para a condição $n>1$. O aspecto marcante de recursividade diz respeito aos dois termos antecessores. Por outro lado, podemos avaliar, na primeira fórmula, que: $n=1 \therefore f_{1}=f_{0}+f_{-1}$. Dai, poderemos ainda obter que $f_{-1}=f_{1}-f_{0}$, todavia, não conhecemos ainda os valores numéricos correspondentes aos termos indicados por $f_{0}$ e $f_{-1}$. Seguindo o mesmo raciocínio, escreveremos $n=2 \therefore f_{2}=f_{1}+f_{0}$ e, dai, inferimos que $f_{0}=f_{2}-f_{1}$. Sabemos que uma sequência em $I R$ é definida por $x_{n}: N \rightarrow N$
$n \mapsto x_{n}$ recursivo, caracterizada por:

$$
\left(f_{N}\right)_{n \in N}:(1 ; 1 ; 2 ; 3 ; 5 ; 8 ; 13 ; 21 ; 34 ; 55 ; 89 ; 144 ; 233 ; 377 ; \ldots)(*) .
$$

Tal sequência é chamada de Sequência de Fibonacci - SF. O termo Fibonacci é a abreviação de filho de Bonaccio, seu pai, como explica Dunlap. Posamentier \&

\footnotetext{
${ }^{1}$ fregis@ifce.edu.br.
} 
Lehmann (2007, p. 22) comentam que "Fibonacci acumulou experiência nos campo da Aritmética e da Álgebra, a partir das viagens que realizou na Europa”, entretanto, apesar de ter desenvolvido vários trabalhos nestes campos da Matemática, Leonardo de Pisa é lembrado geralmente em razão do seu problema que descreve "a reprodução dos coelhos imortais" (GULLBERG, 1997; WELLS, 2005. p. 101), como indicamos abaixo.

Figura 1 - Gullberg (1997) descreve a sequência de coelhos imortais.

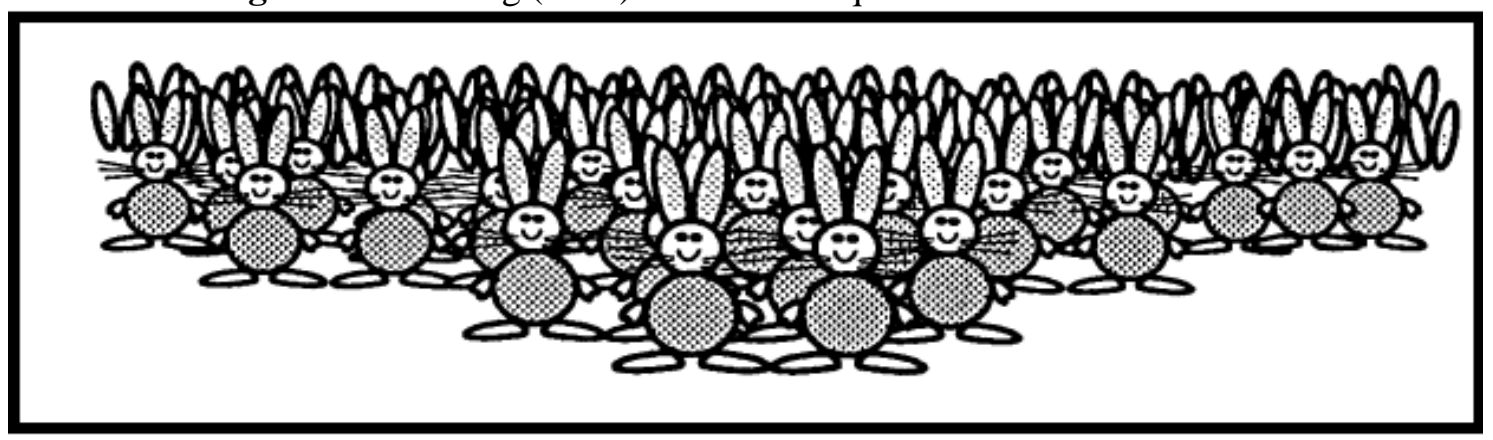

Fonte: Gullberg (1997, p. 286).

Domingues (1991, p. 74) explica que, "provavelmente, para amenizar leitura do Livro do Ábaco, ou torná-la mais interessante", Fibonacci incluiu no livro alguns problemas curiosos e estimulantes, dentre os quais, um veio a se tornar especial: "Um homem põe um casal de coelhos dentro de um cercado. Quantos pares de coelhos serão produzidos num ano, se a natureza desses coelhos é tal que a cada mês um casal gera um novo casal, que se torna produtivo a partir do segundo mês?”.

O questionamento anterior é recorrente e bem divulgado num contexto de cultura de graduação em Matemática, não obstante, a noção e propriedades que buscaremos discutir, nomeada de Sequência Generalizada de Fibonacci - SGF, ainda não ocupa seu devido lugar de destaque. Dessa maneira, na próxima seção, trazemos ao conhecimento do leitor, outras sequências recursivas. Em alguns de nossos trabalhos (ALVES \& BORGES NETO, 2011; ALVES, 2013; 2015), discutimos a generalização do modelo $\left(f_{N}\right)_{n \in N}$ que envolve sua definição no campo dos inteiros. Torna-se natural, então, conjecturarmos que relações podemos obter, na medida em que aumentamos a quantidade de termos antecedentes, com o intuito de definir/descrever outras sequências lineares recursivas, semelhantemente ao caso da SF.

\section{A sequência de Fibonacci e a Tribonacci}


Sem mais delongas, a partir da fórmula de recursividade conhecida, sabemos que: $f_{n+1}=f_{n}+f_{n-1} \therefore \frac{f_{n+1}}{f_{n}}=1+\frac{f_{n-1}}{f_{n}}$. Seguindo um raciocínio semelhante ao que encontramos em Koshy (2011, p. 240), conjecturamos o comportamento de convergência dos quocientes do tipo $\frac{f_{n+1}}{f_{n}}>0$. Desse modo, estabelecemos ainda que: $\frac{f_{n+1}}{f_{n}}=1+\frac{f_{n-1}}{f_{n}} \leftrightarrow \frac{f_{n+1}}{f_{n}}=1+\frac{1}{f_{n} / f_{n-1}}$. Ora, chamando $\quad x_{n+1}:=\frac{f_{n+1}}{f_{n}} \rightarrow x_{n}=\frac{f_{n}}{f_{n-1}} \quad \mathrm{e}$ determinamos a seguinte equação $x_{n+1}=1+\frac{1}{x_{n}} \mathrm{e}$, passando o limite, escreveremos ainda que: $\lim _{n \rightarrow+\infty} x_{n+1}=1+\frac{1}{\lim _{n \rightarrow+\infty} x_{n}} \therefore x=1+\frac{1}{x} \leftrightarrow x^{2}-x-1=0$. Koshy $(2011$, p. 240) declara $\mathrm{o}$ fato conhecido nos livros de História da Matemática que, a raiz positiva da equação $x^{2}-x-1=0$ fornece, justamente, o número de ouro, que é expresso por $\phi=(1+\sqrt{5}) / 2$.

De acordo com a definição, escrevemos: $p_{n=1}=p_{n}+p_{n-1}+p_{n-2} \leftrightarrow\left(\frac{p_{n+1}}{p_{n-1}}\right)=\frac{p_{n}}{p_{n-1}}+1+\frac{p_{n-2}}{p_{n-1}}$. E, seguida, semelhantemente ao expediente usado em Feinberg (1963, p. $72 \quad-\quad 73)$, definimos: $\frac{p_{n+1}}{p_{n}}=t_{n} ; \frac{p_{n}}{p_{n-1}}=t_{n-1} ; \frac{p_{n-1}}{p_{n-2}}=t_{n-2} . \quad$ Daí, teremos ainda que $\frac{p_{n+1}}{p_{n-1}}=\frac{p_{n+1}}{p_{n-1}} \cdot \frac{p_{n}}{p_{n}}=\frac{p_{n+1}}{p_{n}} \cdot \frac{p_{n}}{p_{n-1}}=t_{n} \cdot t_{n-1}$. Desse $\quad$ modo, estabelecemos: $t_{n} \cdot t_{n-1}=t_{n-1}+1+\frac{1}{t_{n-2}} \leftrightarrow t_{n}=1+\frac{1}{t_{n-1}}+\frac{1}{t_{n-1} \cdot t_{n-2}}$. Feinberg (1963, p. 73) admite a convergência da sequência há pouco definida por $\left\{t_{n}\right\}_{n \in I N}$ digamos, para um valor ' $\mathrm{x}$ ', então concluímos: $\quad t_{n}=1+\frac{1}{t_{n-1}}+\frac{1}{t_{n-1} \cdot t_{n-2}} \rightarrow x=1+\frac{1}{x}+\frac{1}{x^{2}} \leftrightarrow x^{3}-x^{2}-x-1=0$. Portanto, encontramos uma equação semelhante ao caso da SF mas, no primeiro caso, encontramos um valor positivo que, na literatura de um curso de graduação, é nominado de número de ouro. Por outro lado, com origem nas fórmulas de Cardano, podemos expressar a raiz real da equação $x^{3}-x^{2}-x-1=0$, da seguinte forma 
$\phi_{3}=(1+\sqrt[3]{19-3 \sqrt{33}}+\sqrt[3]{19+3 \sqrt{33}}) / 3=1.839286755214 \ldots \in$ IR . Pelo que vimos, o número

$\phi_{3}$ possui propriedades semelhantes ao número de ouro, originado de uma equação.

\section{A SEQUENCIA DE TETRANACCI, PENTANACCI, HEXABONACCI E} ETC...

No caso de uma sequência recorrente definida por $q_{n}=q_{n-1}+q_{n-2}+q_{n-3}+\mathrm{q}_{n-4}, n \geq 4$, repetiremos o argumento de Feinberg (1963). Neste sentido,

escrevemos:

$q_{n}=q_{n-1}+q_{n-2}+q_{n-3}+\mathrm{q}_{n-4} \leftrightarrow \frac{q_{n}}{q_{n-3}}=\frac{q_{n-1}}{q_{n-3}}+\frac{q_{n-2}}{q_{n-3}}+1+\frac{\mathrm{q}_{n-4}}{q_{n-3}}$. Podemos ainda escrever: $\quad \frac{q_{n}}{q_{n-3}} \cdot \frac{q_{n-2}}{q_{n-2}} \cdot \frac{q_{n-1}}{q_{n-1}}=\frac{q_{n}}{q_{n-1}} \cdot \frac{q_{n-1}}{q_{n-2}} \cdot \frac{q_{n-2}}{q_{n-3}}=\frac{q_{n-1}}{q_{n-2}} \cdot \frac{q_{n-2}}{q_{n-3}}+\frac{q_{n-2}}{q_{n-3}}+1+\frac{1}{\frac{q_{n-3}}{q_{n-4}}} . \quad$ Nestas expressões, vamos assumir que: $t_{n}=\frac{q_{n}}{q_{n-1}}, t_{n-1}=\frac{q_{n-1}}{q_{n-2}}, t_{n-2}=\frac{q_{n-2}}{q_{n-3}}$ e reescrevemos: $t_{n} \cdot t_{n-1} \cdot t_{n-2}=t_{n-1} \cdot t_{n-2}+t_{n-2}+1+\frac{1}{t_{n-3}}$. Mais uma vez, Feinberg (1963, p. 74) comenta que o quociente acima, obtido a partir da sequência $\left(q_{n}\right)_{n \in I N}$, apresenta um comportamento de convergência, semelhante ao caso encontrado com a SF. Feinberg indica o seguinte valor real $1,9275619 \mathrm{~K}$ Ora, podemos considerar $\lim _{n \rightarrow+\infty} t_{n}=t \therefore \lim _{n \rightarrow+\infty}\left(t_{n} \cdot t_{n-1} \cdot t_{n-2}\right)=\lim _{n \rightarrow+\infty}\left(t_{n-1} \cdot t_{n-2}+t_{n-2}+1+\frac{1}{t_{n-3}}\right)$ e, efetuando as passagens operacionais com limite, inferimos que: $t^{3}=t^{2}+t^{2}+1+\frac{1}{t} \leftrightarrow t^{4}-t^{3}-t^{2}-t-1=0$. Assim, encontramos mais uma vez a seguinte equação polinomial $x^{4}-x^{3}-x^{2}-x-1=0$ relacionada com a sequência recursiva, definida a partir de seus quatros termos antecedentes, que possui como uma das raízes reais, o número indicado por $\phi_{4}$. 


$$
\begin{gathered}
\text { Vamos, agora, expressar a seguinte relação analítica } \\
r_{n}=r_{n-1}+r_{n-2}+r_{n-3}+r_{n-4}+\mathrm{r}_{n-5} \therefore \frac{r_{n}}{r_{n-4}}=\frac{r_{n-1}}{r_{n-4}}+\frac{r_{n-2}}{r_{n-4}}+\frac{r_{n-3}}{r_{n-4}}+1+\frac{r_{n-5}}{r_{n-4}} \text {. Segue ainda que: } \\
\frac{r_{n}}{r_{n-4}} \cdot \frac{r_{n-3}}{r_{n-3}} \cdot \frac{r_{n-2}}{r_{n-2}} \cdot \frac{r_{n-1}}{r_{n-1}}=\frac{r_{n}}{r_{n-1}} \cdot \frac{r_{n-1}}{r_{n-2}} \cdot \frac{r_{n-2}}{r_{n-3}} \cdot \frac{r_{n-3}}{r_{n-4}}=\frac{r_{n-1}}{r_{n-4}} \cdot \frac{r_{n-2}}{r_{n-2}} \cdot \frac{r_{n-3}}{r_{n-3}}+\frac{r_{n-2}}{r_{n-4}} \cdot \frac{r_{n-3}}{r_{n-3}}+\frac{r_{n-3}}{r_{n-4}}+1+\frac{1}{\frac{r_{n-4}}{r_{n-5}}} \therefore \\
\frac{r_{n}}{r_{n-1}} \cdot \frac{r_{n-1}}{r_{n-2}} \cdot \frac{r_{n-2}}{r_{n-3}} \cdot \frac{r_{n-3}}{r_{n-4}}=\frac{r_{n-1}}{r_{n-2}} \cdot \frac{r_{n-2}}{r_{n-3}} \cdot \frac{r_{n-3}}{r_{n-4}}+\frac{r_{n-2}}{r_{n-3}} \cdot \frac{r_{n-3}}{r_{n-4}}+\frac{r_{n-3}}{r_{n-4}}+1+\frac{1}{\frac{r_{n-4}}{r_{n-5}}} \text { Neste ponto, }
\end{gathered}
$$

repetimos o seguinte procedimento: $q_{n}:=\frac{r_{n}}{r_{n-1}} ; \mathrm{q}_{n-1}:=\frac{r_{n-1}}{r_{n-2}} ; q_{n-2}:=\frac{r_{n-2}}{r_{n-3}} ; q_{n-3}:=\frac{r_{n-3}}{r_{n-4}}$ o que resulta em: $\quad q_{n} \cdot q_{n-1} \cdot q_{n-2} \cdot q_{n-3}=q_{n-1} \cdot q_{n-2} \cdot q_{n-3}+q_{n-2} \cdot q_{n-3}+q_{n-3}+1+\frac{1}{q_{n-4}} . \quad$ Para finalizar, assumindo que a convergência da sequência definida pelos quocientes anteriores, escrevemos:

$$
\lim _{n \rightarrow+\infty} q_{n}=q \therefore \lim _{n \rightarrow+\infty}\left(q_{n} \cdot q_{n-1} \cdot q_{n-2} \cdot q_{n-3}\right)=\lim _{n \rightarrow+\infty}\left(q_{n-1} \cdot q_{n-2} \cdot q_{n-3}+q_{n-2} \cdot q_{n-3}+q_{n-3}+1+\frac{1}{q_{n-4}}\right) .
$$

Obteremos, pois, que: $q^{4}=q^{3}+q^{2}+q+1+\frac{1}{q} \leftrightarrow q^{5}-q^{4}-q^{3}-q^{2}-q-1=0 . \quad$ Para concluir, vamos considerar a seguinte sequência recursiva $s_{n}=s_{n-1}+s_{n-2}+s_{n-3}+s_{n-4}+s_{n-5}+s_{n-6}$, para $n \geq 6$. Repetiremos, pois, o processo anterior, escrevendo:

$$
\frac{s_{n}}{s_{n-5}}=\frac{s_{n-1}}{s_{n-5}}+\frac{s_{n-2}}{s_{n-5}}+\frac{s_{n-3}}{s_{n-5}}+\frac{s_{n-4}}{s_{n-5}}+1+\frac{s_{n-6}}{s_{n-5}} \therefore \frac{s_{n}}{s_{n-5}} \cdot \frac{s_{n-4}}{s_{n-4}} \cdot \frac{s_{n-3}}{s_{n-3}} \cdot \frac{s_{n-2}}{s_{n-2}} \cdot \frac{s_{n-1}}{s_{n-1}}=\frac{s_{n-1}}{s_{n-5}}+\frac{s_{n-2}}{s_{n-5}}+\frac{s_{n-3}}{s_{n-5}}+\frac{s_{n-4}}{s_{n-5}}+1+\frac{s_{n-6}}{s_{n-5}}
$$

Em seguida, reescrevemos: $\frac{s_{n}}{s_{n-1}} \cdot \frac{s_{n-1}}{s_{n-2}} \cdot \frac{s_{n-2}}{s_{n-3}} \cdot \frac{s_{n-3}}{s_{n-4}} \cdot \frac{s_{n-4}}{s_{n-5}}=\frac{s_{n-1}}{s_{n-5}}+\frac{s_{n-2}}{s_{n-5}}+\frac{s_{n-3}}{s_{n-5}}+\frac{s_{n-4}}{s_{n-5}}+1+\frac{1}{\frac{s_{n-5}}{s_{n-6}}}$.

Mais uma vez, adotamos a substituição: $h_{n}=\frac{s_{n}}{s_{n-1}} ; h_{n-1}=\frac{s_{n-1}}{s_{n-2}} ; h_{n-2}=\frac{s_{n-2}}{s_{n-3}} ; h_{n-3}=\frac{s_{n-3}}{s_{n-4}} ; h_{n-4}=\frac{s_{n-4}}{s_{n-5}}$ o que conduz a nova igualdade: $h_{n} \cdot h_{n-1} \cdot h_{n-2} \cdot h_{n-3} \cdot h_{n-4}=h_{n-1} \cdot h_{n-2} \cdot h_{n-3} \cdot h_{n-4}+h_{n-2} \cdot h_{n-3} \cdot h_{n-4}+h_{n-3} \cdot h_{n-4}+1+\frac{1}{h_{n-5}}$. Ora, com arrimo nos argumentos anteriores, encontramos a seguinte equação $h^{6}-h^{5}-h^{4}-h^{3}-h^{2}-h-1=0$ que possui, como umas das raízes reais positivas, o número 
$\phi_{6}$. Encerramos a presente seção assinalando que as últimas argumentações, de modo geral, são desconsideradas e não pormenorizadas por autores de livros de História.

\section{Considerações finais}

Na seção anterior, de acordo com referências especializadas e pouco divulgadas no locus acadêmico (VOROBE`V, 1974), discutimos propriedades relacionadas com as sequências de tribonacci, tetranacci, pentanacci e hexabonacci. Ora, com arrimo nos argumentos anteriores, podemos conjecturar a existência de outras propriedades originadas da SF e adquirir um entendimento da evolução histórica de um modelo que se mostra indene aos efeitos temporais, séculos e séculos após sua proposição. Apresentamos, pois, equações polinomiais que resultam no número de ouro $\phi$, bem como outros números derivados, que denotaremos por $\phi_{3}, \phi_{4}, \phi_{5}, \phi_{6}, \mathrm{~K}, \phi_{n}, \mathrm{~K}$ (FENG, 2011).

\section{Referências}

ALVES, F.R.V. Sobre a evolução histórica do modelo de Fibonacci: a classe das funções hiperbólicas de Fibonacci. Revista Vydia Educação. v. 35, nº , 133 - 148, 2015. [online] < http://periodicos.unifra.br/index.php/VIDYA $>$.

ALVES, F.R.V. Uma discussão de artigos envolvendo propriedades da Sequência de Fibonacci apoiada na Tecnologia. Anais do VI HTEM. 1 - 17, 2013. [online] <http://htem2013.dm.ufscar.br/anais/anais_do_vi_htem_ufscar_2013.html>

ALVES, F.R.V; BORGES NETO, H. A existência de sequência de Fibonacci no campo dos inteiros: uma atividade de investigação apoiada nos pressupostos da Sequência Fedathi. BOLETIM GEPEM. $\mathrm{n}^{\mathrm{o}}$ 59, $135-140,2011$. [online] $<$ http://www.ufrrj.br/SEER/index.php?journal=gepem\&page=article\&op=view\&path\% $\underline{5 \mathrm{~B} \% 5 \mathrm{D}=647>}$

DOMINGUES. Hygino. H. Fundamentos de Artimética. São Paulo: Editora Atual, 1991.

FEINBERG, M. Fibonacci-Tribonacci. The Fibonacci Quartely. v. 1, no 3, October, 209 - 222. 1963. [online] < http://www.fq.math.ca/Scanned/1-3/feinberg.pdf>

FENG, J. More identities on the Fibonacci Numbers. Ars Combinatoria. $n^{\circ} 100,73-$ 78, 2011. 
GULLBERG, Jan. Mathematics: from the birth of numbers. New York: W. W. Norton \& Company, 1997.

KOSHY, T. Fibonacci and Lucas Numbers with Applications. New York: John Wiley and Sons, 2011.

POSAMENTIER, Alfred. S. \& LEHMANN, Ingmar. The fabulous fibonacci numbers. New York Prometeus Book. 2007.

VOROBE`V, N. N. Números de Fibonacci. Leciones Populares de Matemática. Moscou: Editora MIR, 1974.

WELLS, David. Prime Numbers: the mysterious figures in the Math. New Jersey: John Wiley and Sons. Inc. 2005. 